\title{
Successful management of cervical ectopic pregnancy following invitro fertilisation
}

\section{Aloy Okechukwu Ugwu ${ }^{1}$, Christian Chigozie Makwe ${ }^{1,2}$, Sunday Isaac Omisakin ${ }^{1,2}$, Nneoma Kwemtochukwu Ani-ugwu'3 , Egba Augustine', Lulu Gethrude Ojiefoh², Ifunanya Tobechukwu Okafor ${ }^{2}$}

\author{
'Department of obstetrics and gynaecology, Lagos University Teaching Hospital \\ ${ }^{2}$ College of medicine, university of Lagos \\ ${ }^{3}$ Department of medicine, Lagos University Teaching Hospital
}

\section{Corresponding Author}

Aloy Okechukwu Ugwu, Senior Registrar, Department of Obstetrics and Gynaecology, Lagos University Teaching Hospital, P.M.B. 12003, Surulere Lagos, Nigeria. Tel.: +2347033132189, e-mail.: okeyugwu92@gmail.com

\section{Abstract}

We report a case of a 45-year-old woman with successful management of cervical ectopic pregnancy using ultrasound guided suction evacuation and balloon tamponade. The case report owes its significance to the high morbidity and mortality associated with this form of ectopic pregnancy in our environment, because of misdiagnosis and late presentation.

Key words: Cervical ectopic pregnancy, invitro-fertilisation, surgical management, Lagos, Nigeria

\section{Introduction}

Cervical ectopic pregnancy is a very rare form of extrauterine pregnancy in which the fertilized ovum implants in the lining of the endocervical canal ${ }^{1}$. It has also been described as pregnancies below the internal cervical os ${ }^{1,2}$. It contributes for less than one percent of extrauterine pregnancies ${ }^{1,2}$. It's an important form of ectopic pregnancy as it poses a serious threat to maternal life if undiagnosed or if not properly managed $^{2}$. Many risk factors for this form of ectopic pregnancy has been suggested to include- smoking, previous history of poorly treated pelvic inflammatory diseases, puerperal sepsis, previous ectopic pregnancy, precious uterine surgeries such as caesarean section, previous myomectomy, polypectomy, metroplasty and dilatation and curettage. Other documented risk factors include intrauterine contraceptive device, congenital anomalies of the female genital tract and invitro fertilization and embryo transfer ${ }^{2-5}$.

Women with cervical ectopic pregnancies may be asymptomatic, or may present with life-threatening haemorrhage. Other forms of presentation may be soft, disproportionately large or barrel-shaped cervix ${ }^{4,5}$. The treatment option depends on clinical presentation and haemodynamic stability of the woman, expertise available and patient's choice or prefrence $^{2,4}$. These options include single or multi- 
dose methotrexate, intramniotic injection of potassium chloride, dilation and curettage, ultrasound guided aspiration with balloon tamponade insertion, uterine artery embolization, laparoscopic exploration and total hysterectomy in extreme cases ${ }^{5,6}$.

\section{Case Report}

Mrs. EN is a 45 -year-old G3P ${ }_{1}^{+1}$ female with seventeen years' history of infertility with two previous uterine surgeries (caesarean section and myomectomy). She presented to our facility for assisted conception. Her last menstrual period was $2^{\text {nd }}$ of July 2021, and she had an heterologous invitro fertilisation cycle with long agonist protocol. She had an easy day- 5 blastocyst transfer on $25^{\text {th }}$ August 2021 .

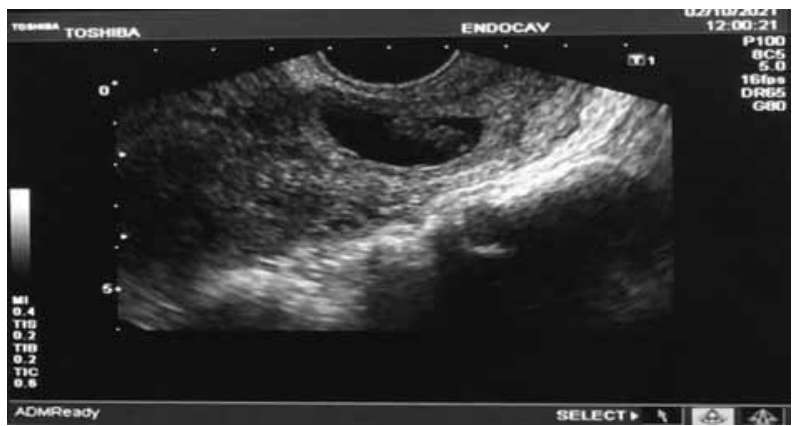

Figure 1.

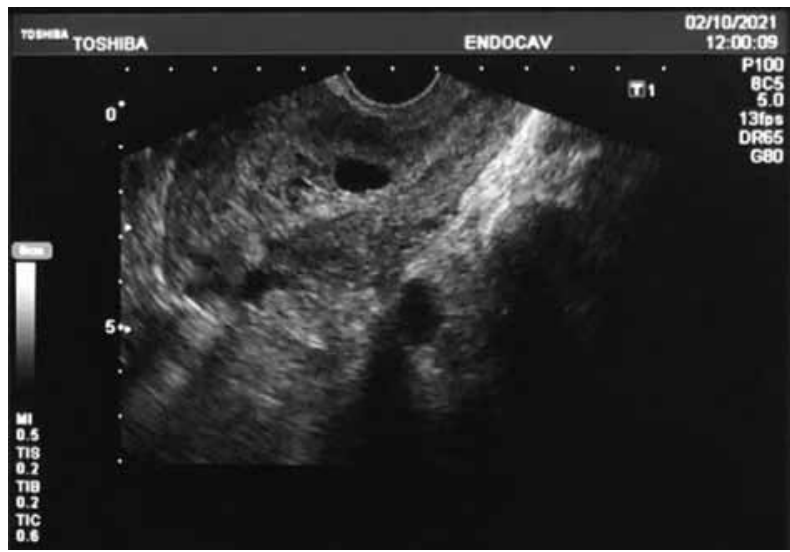

Figure 2.
Pregnancy was detected with a positive serum beta human chorionic gonadotropin on $8^{\text {th }}$ September 2021. She was scheduled for routine pelvic ultrasonography for confirmation of clinical pregnancy on $29^{\text {th }}$ of September 2021; and the scan showed an empty endometrial cavity with an endometrial plate thickness of $10.1 \mathrm{~mm}$. There were no masses on both adnexae. There was a ballooned-out cervical canal that contained a regular gestational sac (see Figures 1, 2 and 3). The gestational sac contained a yolk sac and a fetal pole with evidence of fetal cardiac activity. The embryo's crown rump length was consistent with a seven weeks' gestation. The internal cervical os was closed and located just distal to the gestational sac. She was subsequently counselled on the diagnosis and the need to urgently discontinue the pregnancy. She opted for ultrasound guided evacuation and insertion of balloon tamponade. An informed written consent was obtained for the procedure and an additional consent for hysterectomy due to the possibility of uncontrollable torrential haemorrhage. The appropriate baseline investigations were done and compatible blood was grouped and saved. The transvaginal evacuation of the cervical ectopic pregnancy under transabdominal ultrasonography guidance was performed under spinal anaesthesia.

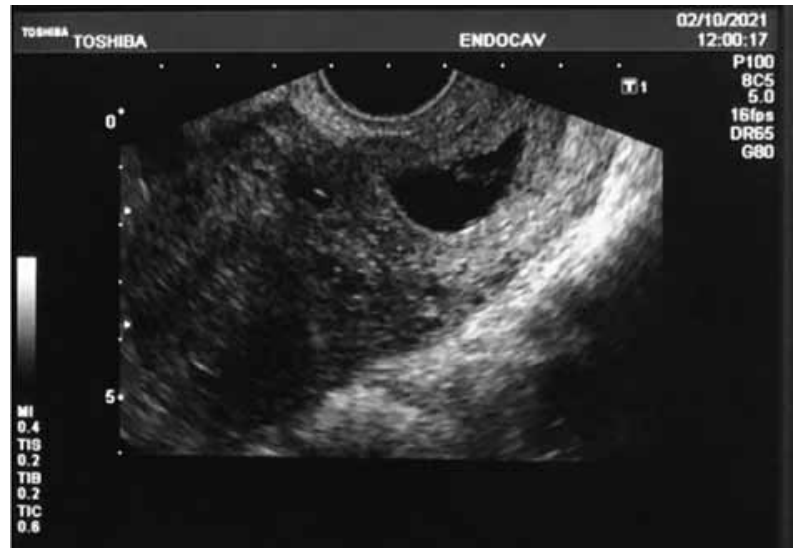

Figure 3. 
A prophylactic Foley catheter (size 18 French gauge) was inserted into the cavity and the balloon inflated with 20 of sterile normal saline. Intra-operatively, her vital signs remained stable and the estimated blood loss was $200 \mathrm{~mL}$. Postoperatively, she was given prophylactic antibiotics and oral analgesics. The Foley catheter was removed after 24 hours and there was no reactionary haemorrhage. Mrs. EN had good clinical recovery and was discharged home after 30 hours' observation in hospital. Her two weeks follow-up visit was essentially normal. The specimen (product of conception) was sent for histopathological analysis, which also confirmed the diagnosis.

\section{Discussion}

The significance of cervical ectopic pregnancy cannot be overemphasized been the second commonest site of non-tubal ectopic pregnancy preceded by the ovarian ectopic gestation ${ }^{2,5}$. The overall occurrence of this form of ectopic pregnancy seems to be rising due to increased utilisation of different forms of assisted conception ${ }^{4,5}$. The risk factors found in Mrs. EN include, advancing maternal age, previous uterine surgeries- myomectomy, polypectomy and previous caesarean section and use of assisted conception.

Recently, there has been a reduction in the difficulties associated with the diagnosis of cervical ectopic pregnancy due to wide availability of high-resolution ultrasonography. Several criteria abound in literature for the diagnosis of cervical ectopic pregnancy ${ }^{5,6}$. These criteria include both radiological and pathological findings ${ }^{2,6}$. Radiological findings include an empty uterine cavity with a barrel-shaped cervix on ultrasound, regular gestational sac inferior to the internal cervical os, intact cervical canal between the endometrium and gestational sac, absence of the 'sliding sign', and demonstration of blood flow around the gestational sac using colour Doppler; while the pathological criteria include presence of cervical glands adjacent the placental attachment, placenta situated below the entrance of uterine vessels, or inferior to the peritoneal reflection of the anterior and posterior surface of the uterus ${ }^{5-8}$.

Our patient had a successful treatment of an un-ruptured cervical ectopic pregnancy. Her early presentation and diagnosis were partly due to the routine practice of early ultrasonography following invitro fertilization, the use of high-resolution transvaginal ultrasonography in the confirmation of clinical pregnancy, and the skill and expertise of the sonographers. Although the cervical ectopic pregnancy was un-ruptured and asymptomatic, her condition was considered urgent because of the potential of life-threatening haemorrhage following rupture of the ectopic. She had an uncomplicated evacuation of cervical ectopic aided by Foley catheter balloon insertion.

\section{References}

1. Arowojolu AO, Ogunbode 00. Cervical ectopic pregnancy managed with methotrexate and tranexamic acid: A case report. Afr. J. Med. med. Sci. 2014; (43): 361-4

2. Olamijulo JA, Okusanya BO, Adenekan MA, Ugwu AO, Olorunfemi G, Okojie O. Ectopic pregnancy at the Lagos University Teaching Hospital, Lagos, South-Western Nigeria: Temporal trends, clinical presentation and management outcomes from 2005 to 2014. Niger Postgrad Med J 2020; 27: 177-83.

3. Takeda K, Mackay J, Watts S. Successful management of cervical ectopic pregnancy with bilateral uterine artery embolization and methotrexate. Case Rep Emerg Med. 2018: 1-4

4. Matorras R, Zallo A, Hernandez-Pailos R, Ferrando M, Quintana F, Remohi J. et al. Cervical pregnancy in assisted reproduction: an analysis of risk factors in 91,067 ongoing pregnancies. Reprod Biomed Online 2020; 40:355.

5. Hwang JH, Lee JK, Oh MJ, Lee NW, Hur JY, Lee KW. 
Classification and management of cervical ectopic pregnancies: experience at a single institution. J Reprod Med. 2010 Nov-Dec;55(11-12):469-76.

6. Awowole IO, Adeniyi OA, Allen 00, Adeyemi AB. Fertility-preserving Management of Cervical Ectopic Pregnancy in a Nigerian Teaching Hospital. Annals of Health Research. 2021: 7(3): 322-7.

7. Samal SK, Rathod S. Cervical ectopic pregnancy. J Nat Sci Biol Med. 2015;6(1):257-60.
8. Kirk E, Condous G, Haider Z, Syed A, Ojha K. Bourne T. The conservative management of cervical ectopic pregnancies. Ultrasound Obstet Gynecol 2006; 27: 430-7.

Received 15-09-21

Revised 23-09-21

Accepted 30-09-21 